Meta

Journal des traducteurs

Translators' Journal

\title{
Divers déplacements de sens dans les anglicismes sémantiques
}

\section{Gilles Colpron}

Volume 15, numéro 1, mars 1970

La traduction religieuse

URI : https://id.erudit.org/iderudit/002353ar

DOI : https://doi.org/10.7202/002353ar

Aller au sommaire du numéro

Éditeur(s)

Les Presses de l'Université de Montréal

ISSN

0026-0452 (imprimé)

1492-1421 (numérique)

Découvrir la revue

Citer cet article

Colpron, G. (1970). Divers déplacements de sens dans les anglicismes

sémantiques. Meta, 15(1), 36-39. https://doi.org/10.7202/002353ar d'utilisation que vous pouvez consulter en ligne.

https://apropos.erudit.org/fr/usagers/politique-dutilisation/ 


\section{DIVERS DÉPLACEMENTS DE SENS DANS LES ANGLICISMES SÉMANTIQUES}

La plupart des anglicismes de sens consistent simplement en un emploi qui s'écarte de l'aire sémantique consacrée du mot utilisé. Ainsi, si on emploie le mot «accommodation» au sens de logement, on étend l'aire sémantique de ce mot, on emploie celui-ci dans un sens extérieur à son aire sémantique reconnue. Mais à part ces significations simplement étendues, ou ces acceptions extérieures à l'aire sémantique reconnue - qui nous laissent sur un plan « horizontal » il y a aussi des emplois qui, sans se situer en dehors des virtualités sémantiques du mot, présentent un déplacement en quelque sorte vertical de sa signification.

Ce genre de déplacement prend plusieurs formes, parmi lesquelles on peut citer d'abord le phénomène de précision, ou de particularisation, d'un terme qui en français n'a qu'un sens général. Il n'est sans doute pas faux de qualifier de «sièges » les fauteuils d'une salle de théâtre ou de cinéma, puisque «siège » est le terme général qui désigne tout «meuble ou autre objet fait pour s'asseoir » (Larousse). Néanmoins, l'usage veut qu'on emploie un terme particulier, qu'on appelle couramment fauteuils les sièges ordinaires d'une salle de cinéma ou de

7. H. Piraux, Dictionnaire anglais-français des termes relatifs à l'électrotechnique et l'électronique, $4^{\mathrm{e}}$ éd., Paris, Eyrolles, 1959.

Voir aussi G. G. King, Dictionnaire anglais-français, électronique et physique nucléaire, Paris, Dunod, 1959. 
théâtre, banquettes ceux d'un autobus ou d'un train, gradins ceux d'un amphithéâtre et selle le siège que comporte une bicyclette. C'est imiter l'anglais, qui désigne couramment par seat toutes ces sortes particulières de siège, que d'employer dans chacun de ces cas le terme générique à la place du terme particulier. Il en va de même pour le «séparateur» de nos fermiers canadiens-français. Si leurs collègues canadiens-anglais se contentent du terme générique separator, en français, c'est le mot précis écrémeuse qui s'emploie. Voilà autant de cas où, sous l'influence très probable de l'anglais, on donne à un mot français un sens plus précis qu'il n'a en réalité.

Il y a aussi le phénomène contraire : l'attribution d'un sens général à un terme doté d'une signification très particulière. Le mot «breuvage», dans le français moderne, ne désigne qu'un liquide fortifiant ou curatif donné à boire aux animaux, ou une tisane ou autre boisson médicamenteuse préparée pour un malade. C'est donc une sorte bien particulière de boisson. Mais la masse des Canadiens français, sous l'influence de l'anglais beverage, entend ce vocable dans le sens de boisson en général. C'est ainsi que la télévision nous annonce une boisson gazeuse en la proclamant « le breuvage pétillant». Au restaurant, la serveuse nous demande : «Qu'est-ce que vous allez prendre comme breuvage?» Dans les cantines, les petites boîtes de carton qui contiennent une boisson faite de poudre de chocolat ou de cacao délayée portent l'inscription «Breuvage au chocolat». On donne donc à «breuvage » le sens général de boisson. De même, on donne chez nous à «escompte» le sens général de remise, de rabais, de réduction, qu'a le mot anglais discount, alors qu' « escompte » ne désigne en réalité que la réduction du montant d'une dette à terme lorsque celle-ci est payée avant l'échéance. Ce sont là des exemples d'anglicismes qui représentent un passage du particulier au général.

D'autres anglicismes s'apparentent à ceux-là du fait qu'ils paraissent excusables eux aussi en raison de leur caractère non tout à fait étranger aux aires sémantiques consacrées des mots en question. Ici, l'acception venant de l'anglais, plutôt que de s'écarter du sens français du mot, s'y superpose, ou plus précisément, constitue une espèce de sens figuré, forme une figure de style, une image. Lorsqu'on dit «approcher quelqu'un», au sens de lui parler une première fois de quelque chose, de lui faire la proposition de quelque chose, de le pressentir, c'est un sens imagé que l'on donne au verbe «approcher » : prendre un premier contact plutôt prudent avec l'état d'esprit ou la disposition de telle personne. Il en est ainsi pour «deux opinions irréconciliables ». Même si le strict bon usage français nous commande d'employer plutôt l'adjectif inconciliables, on doit admettre comme assez naturelle cette comparaison d'idées avec des personnes. Le «poisson» (le gogo, la poire) qui se laisse facilement berner et la personne qui « vaut » (possède) cinq millions sont d'autres illustrations de la figure de style à laquelle équivaut parfois l'anglicisme.

D'autre part, certains termes français concrets reçoivent au Québec, sous l'influence de l'anglais, un sens abstrait. C'est le cas de «blanc » dans l'expression «blanc de mémoire ». Dans son sens français, celui d'intervalle, d'espace libre qu'on laisse dans un écrit, blanc est un terme concret. On le transpose dans 
l'abstrait lorsqu'on l'applique à l'esprit en lui faisant désigner une absence ou un trou de mémoire.

Le phénomène inverse se présente aussi. En donnant à «dénomination »le sens d'église (ex. : «catholiques, anglicans, presbytériens et membres des autres dénominations »), on identifie l'appellation, qui est essentiellement quelque chose d'abstrait, avec la chose désignée, l'être concret. Donc, phénomène de concrétisation de sens.

Autre genre de déplacement vertical du sens des mots : la dévalorisation. On dévalorise ou on « diminue », par exemple, le mot «saveur » lorsqu'on l'emploie au sujet d'un parfum de crème glacée ou d'une essence de gelée ou autre préparation alimentaire. La saveur n'est pas une notion neutre, elle est une qualité à un degré élevé; en lui donnant le sens précité (par décalque automatique de l'anglais flavour), on lui enlève sa force qualitative.

L'inverse se fait aussi, par exemple lorsqu'on emploie «compléter» dans le sens d'exécuter. Compléter des travaux, c'est les achever, les rendre complets, terminés; c'est en exécuter la phase finale, donc une seule phase, et non pas toutes. Lorsqu'on annonce l'entreprise de travaux de construction et qu'on dit : "Les travaux seront complétés en deux ans », on fait exprimer au verbe «compléter» une action plus vaste qu'il n'en exprime en vrai français, on «augmente» la réalité que ce terme exprime dans son acception légitime.

L'anglicisme peut équivaloir aussi à donner une valeur active à des termes qui, en réalité, marquent un état ou s'appliquent à l'être passif, à celui qui reçoit l'action plutôt qu'il ne l'exerce. Exemple : l'adjectif «préjugé » employé au sens de "prévenu » (prejudiced). En fait, c'est la question ou la cause soumise qui est préjugée par la personne prévenue. En disant que la personne est «préjugée », on lui attribue un rôle passif, ou bien on donne faussement une valeur active au prédicat qu'on lui accole. Autre illustration : le mot «sécurité » employé dans le sens de «garantie». En français, la sécurité désigne l'état que peut procurer la garantie.

Le phénomène inverse est celui de la «passivation ». C'est le fait, par exemple, d'employer le verbe "goûter » dans le sens d' « avoir goût de », alors que ce verbe exprime l'action de percevoir, d'apprécier par le sens du goût la saveur d'un aliment ou d'une boisson. Le verbe «regarder» subit le même genre de traitement dans la phrase: "Il regarde mieux ce matin 》 (he looks better this morning). Regarder, c'est l'action de celui qui constate que la personne en question paraît mieux, a meilleur air. On donne donc, par un tel emploi, un sens passif au verbe « regarder».

Citons aussi le phénomène de la «transitivation», qui consiste à donner un sens transitif à un verbe exprimant une action strictement intransitive en français. On en a des exemples dans les expressions « courir un cheval (pour faire courir) et «partir un moteur» (pour faire partir ou mettre en marche). Le phénomène contraire est l'《intransitivation », ou l'attribution d'une valeur intransitive à un verbe d'emploi uniquement transitif en français. C'est le cas du tour «Jean a gradué 
cette année », pour « Jean a été gradué * / a obtenu son grade / a obtenu son diplôme ».

Enfin, signalons les cas d' « inversion », comme le fait de dire «j'ai été assigné à cette tâche » plutôt que cette tâche m'a été assignée, «je suis familier avec ce procédé » plutôt que ce procédé m'est familier, « il est sympathique à cette jeune candidate » plutôt que cette jeune candidate lui est sympathique, ou « je vais vous manquer» (I'll miss you) plutôt que vous allez me manquer.

Gilles Colpron

* A noter que seul le Quillet, parmi les dictionnaires les plus connus, donne au verbe " graduer " le sens de conférer un grade.

1. Jean Chevalier, Organisation, 10e éd., Paris, Dunod, 1961, vol. I, p. 39. 